\title{
Image Thresholding using Histogram Fuzzy Approximation
}

\author{
Mohammad A. N. Al-Azawi ${ }^{(1)(2)}$ \\ ${ }^{(1)}$ Oman College of Management and Technology, Barka, Oman \\ ${ }^{(2)}$ Centre for Computational Intelligence, DMU, Leicester, UK
}

\begin{abstract}
Image segmentation is one of the most important techniques in image processing. It is widely used in different applications such as computer vision, digital pattern recognition, robot vision, etc. Histogram was the earliest feature that has been used for isolating objects from their background, it is widely applicable in different application in which one needs to divide the image into distinct regions like background and object. The thresholding technique is the most popular solution in which a value on the histogram is selected to separate the regions. This value, which is known as the threshold, should be specified in an appropriate way. One of the methods is by using the global minimum value of the histogram and divides the histogram into white and black (binary image). Due to the spatial and grey uncertainty and ambiguity, the extraction of the threshold value in a crispy way is not suitable always. To overcome such problems, the proposed method uses two membership functions to measure the whiteness and blackness of a member element. The pixel belonging to one of the region is dependent on the membership value it has according to the membership functions.
\end{abstract}

\section{General Terms}

Pattern Recognition, Artificial intelligence, Image Processing

\section{Keywords}

Image segmentation, bimodal histogram, fuzzy intelligence, thresholding

\section{INTRODUCTION}

Image processing is an important field of computer science and engineering. It is widely applicable in different applications such as computer vision, robotic, pattern recognition, etc. Image vision and understanding is one the most important challenge in this field. Dividing scenes into distinct regions that go through the recognition and understanding process is a crucial issue in image understanding. The division or splitting process is known as the segmentation process. Many algorithms have been proposed to improve the performance of such algorithms. There are many applications for this process in different fields, such as, medical applications, industrial applications, machine vision, products quality assurance in factories, pattern recognition, and many others.

Artificial intelligence is widely used in image processing applications and image recognitions and understanding. Techniques such as Genetic Algorithms, Neural Networks, and Fuzzy Logic are used to improve the results obtained from different image processing techniques. The uncertainties in grey values and in spatial space are great motivations to use fuzzy logic in image processing.

This paper presents an algorithm that utilizes the histogram thresholding technique using fuzzy logic theory in image segmentation. Histogram gives good information about the distribution of the grey levels over pixels. Since most of the algorithms assume the input image as a dark object in a light background, or vice versa, thus the histogram is expected to have two peaks and one valley. The valley, which represents the minimum point between the two peaks, is considered as the threshold value. This thresholding technique is known as bimodal thresholding. Due to the fact that, there are no distinct borders between the object and the background because of the imaging and lighting properties, thus it is not feasible to isolate the object from its background using crispy methods. Most of the literatures are focusing on how to find the value of the threshold value, which is the grey value that separates the object from its background.

The rest of the paper shall include the necessary theoretical background and review of the available techniques in section (2). Section (3) will describe the proposed technique and the theoretical derivation. In section (4) testing and experimental results are listed, and finally in section (5) the conclusions are derived.

\section{BACKGROUND AND REVIEW}

Many models of image representations are used to represent the image in digital format, formats such as RGB, HSL, VSL, and other models are used to represent the coloured images [1]. The most widely used model in image processing is greylevel image representation model. In this model the image $I(x, y)$ is represented by the luminance $I$ of the pixels at the spatial location $x$ and $y$. The number of grey levels (luminance levels) is equal to $2^{\mathrm{n}}$, with $n$ is the number of bits that are used to represent each pixel value. The most commonly used representation is by representing each pixel by 8 bits (byte) which results in having a total of 256 grey levels.

Image processing techniques are applied to change the luminance values according to some criteria, applications like image sharpening (High Pass Filters), smoothing (Low Pass Filters), enhancement, restoration, contrast enhancement, etc. can be used to improve the image and to extract some important features that can be used in image recognition and understanding. Figure 1 shows a simple representation of an image processing system. The system gets a raw image and apply different enhancement and image processing techniques and the output will be an enhanced, well-recognized image; this process can be represented in the fallowing equation:

$$
G(x, y)=F(I(x, y))
$$

Where $G(x, y)$ is the processed image resulted from applying the processing $F($.$) on the original image$ 


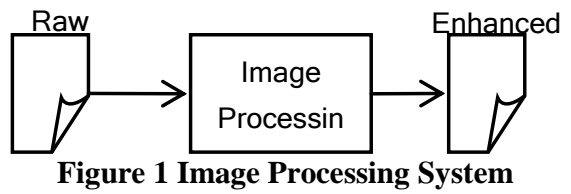

\subsection{Image Histogram and Features}

For an image $I(x, y)$ with size of $N \times M$ and number of grey levels of $\mathrm{n}$, the histogram can be expressed as:

$$
\vec{H}=<h_{1}, h_{2}, h_{3}, \ldots . h_{n},>
$$

Where $\vec{H}$ is the histogram vector and $h_{i} ; i=1,2, \ldots, n$ is number of pixels of colour level $i$ in the image.

Several features and measures can be extracted from histogram to describe the contents of an image like colour moments, such as, mean (first order), variance (second), and skewness (third).

The histogram can be considered as a measure to the information contents in the image. As it was assumed that the image contains two major parts, objects and background, thus, the histogram information contents can be expressed as follows:

$$
\boldsymbol{H}=\boldsymbol{H}_{O}+\boldsymbol{H}_{B}
$$

With $\boldsymbol{H}_{O}$ and $\boldsymbol{H}_{B}$ are the object and background image information contents respectively. The following figure shows the histogram components of the grey histogram.

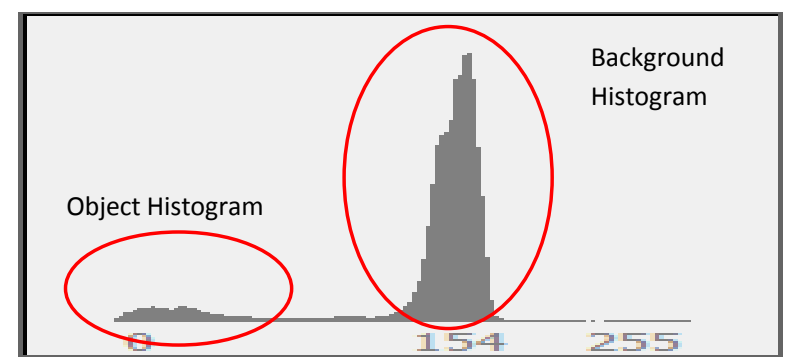

Figure 2 Histogram components

Figure 2 shows the two components of the grey histogram of the image, form the figure it is clear that the background component has higher values for the bins; this will lead to giving the background component more significance than the object component in the features or measure extraction.

For instance let us assume that the colour values in an image are represented by the set $\Omega$, which represents the universal set, such that:

$$
\Omega=\left\{x_{i}: 1 \leq i \leq W \times H\right\}
$$

Where $x_{i}$ is the value of the pixel colour of the image considering that the image pixels are arranged to be a onedimensional vector

The set of the pixels is divided into two components, object $\Omega_{O}$ and background $\Omega_{\mathrm{B}}$ :

$$
\Omega=\Omega_{O} \cup \Omega_{B} ; \Omega_{S}=\left\{x_{O i}\right\} ; \Omega_{B}=\left\{x_{B i}\right\}
$$

If the borders between the object and background are considered to be crispy, which means that the object is isolated from the background and the borders are welldefined, i.e. $\Omega_{O} \cap \Omega_{\mathrm{B}}=\emptyset$ then the cardinality of the set will be as follows:

$$
\begin{gathered}
|\Omega|=\left|\Omega_{O}\right|+\left|\Omega_{B}\right|-\left|\Omega_{O} \cap \Omega_{B}\right| \\
\left|\Omega_{O} \cap \Omega_{B}\right|=0 \\
|\Omega|=\left|\Omega_{O}\right|+\left|\Omega_{B}\right|
\end{gathered}
$$

Based on the definition of the histogram, we shall define the function $H(x)$ as the number of occurrence of the variable $x$ as follows:

$$
H\left(x_{i}\right)=P\left(x_{i}\right)
$$

Where $\mathrm{P}\left(\mathrm{x}_{\mathrm{i}}\right)$ is the probability of occurrence of the bin $x_{i}$. $P\left(x_{i}\right)$ contains the two components mentioned above i.e.:

$$
H\left(x_{i}\right)=H_{B}\left(x_{i}\right)+H_{O}\left(x_{i}\right)
$$

Where $H_{B}\left(x_{i}\right)$ and $H_{O}\left(x_{i}\right)$ are the probability of occurrence of the variable $x_{i}$ in the background and in the object regions respectively.

In equation 6 , the intersection between the object and the background sets $\Omega_{O} \cap \Omega_{\mathrm{B}}$ was ignored which is not the real case; usually there should be intersection between them due to the uncertainty and the shadow. Thus, fuzzy logic is used in defining the border between the regions and the background.

\subsection{Image Segmentation and Thresholding}

Segmentation can be defined as the process of dividing the image into non-overlapping, homogenous, and connected regions [2]. The regions are separated according to some attributes like luminance amplitude for grey images and colour components for colour images [3]. Several other attributes like texture, shapes, and edges are also used in segmentation. Many types of segmentation have been proposed by literature using different techniques like in ref [4]. Thilagamani and Shanthi [5] have published a survey on segmentation through clustering, they have presented different techniques in segmentation, and they define the clustering as the grouping of similar images in the database. Pappas [6] proposed the use of adaptive clustering technique in image segmentation. He suggested a generalized K-Mean algorithm in clustering to include spatial constraints and to account for local intensity variations in the image. Artificial intelligencebased segmentation was used by many authors like Deshmukh and Shinde who have used neuro-fuzzy system for colour image segmentation [7].

Some segmentation algorithms utilize luminance in grey images in which colour images should be converted into grey images before applying these algorithms. While some other techniques consider the colour images in segmentation process which give better results as they are analogy to human perception.

Segmentation algorithms may be classified further as local and global segmentation algorithms. In local segmentation, only the features of the pixels values and their neighbouring pixels are considered, while in global segmentation the image as a whole is considered in the segmentation process [8].

Segmentation divides the image into a set of regions $R$, which consists of homogenous, non-overlapped, connected subregions $R_{i}$, i.e.

$$
R=\left\{R_{i}: i=1,2, \ldots, N\right\}
$$

The union of all subregions forms the original image i.e.

$$
I=R_{1} \cup R_{2} \cup \ldots \cup R_{N}
$$

The regions $R_{i}$ should be connected for all $i=1,2, \ldots, N$ and each regions $R_{i}$ should be homogeneous. Finally, different adjacent regions $R_{i}$ and $R_{j}$ should be disjoint i.e.

$$
R_{i} \cap R_{j}=\emptyset
$$


Fuzzy-based techniques were used as a segmentation process by many authors. Cheng et al. [9] proposed using the cpartition entropy fuzzy to select the threshold value. Maximum entropy principle and fuzzy c-partition method have been used to select the threshold value(s) associated with the maximum entropy of the fuzzy c-partition. They have assumed a fixed and known number of the clusters and classified the pixels into these clusters. Entropic thresholding was also used by Wang et al. [10] Tao et al. [11] and others. Yong, Chongxun, and Pan [12], and Junwei et al. [13], have used c-mean with fuzzy logic as a clustering algorithm in their papers published in 2004 and 2007 respectively. Tizhoosh has suggested the use of ultrafuzzy (fuzzy II) algorithms to find the threshold value [14]. Prasad et al. have suggested a fast unsupervised thresholding algorithm based on $\pi$ membership function [15]. In their method, Prasad et al. have considered a fuzzy $\pi$-function to transform the fuzzy intensities into normally distributed intensities. The intensities of an image are transformed to an interval $[0,1]$ by $\pi$-function in terms of a standard $\mathrm{S}$-function. The values of the function represent the degrees of the closeness in terms of intensities. The function, therefore, is used to locate the intensities of object and background. The selection of a cross-over point which is the arithmetic mean of the image could be viewed as an objectbackground classification problem.

From reviewing the available algorithms, we have identified several problems we shall consider in the proposed algorithm. The most important problem is the computation amount needed in some algorithm because of the iteration they contain, which we are going to overcome in this paper, since the proposed algorithm is not iterative.

\subsection{Fuzzy Intelligence}

Fuzzy logic was invented in 1965 by Zadeh in his research Fuzzy Sets [16]. Zadeh defined the fuzzy sets and fuzzy logic in additional to the membership function which assign each grade to a value between one and zero and introduced the theoretical background. Fuzzy is a representation of uncertainty in which each logic operation like OR, AND, and NOT is represented in a way differs from the True/False binary space. First, it was proposed to have a third value between the binary values (True and False), and then this principle has been generalized to have infinite number of possibilities between them. Assume that $X=\{x\}$ is a set of points and $\mu_{A}(x)$ is the characteristics function or membership function, then, $\mu_{A}(x)$ which measures the membership of $x$ in the class assigns each $x$ into a real value in the interval $[0,1]$. The closer value of $\mu_{A}(x)$ to one, the more properly that $x$ be a member of $A$. All the binary logical operations are defined according to the above definition like OR operation is replaced by MAX, AND is replaced by MIN, and NOT operation is replaced by complement, the detailed theory is found in [16].

Fuzzy sets are extension to the idea of crisp sets in which an element is a member in a class if the value of the membership function is one, and not a member in the class if it is 0 ; According to this definition, an element either a member of the class or not a member at all. Fuzzy sets proposed the partial membership, in which the strength of membership can be found. Partial membership allowed an element to be member in more than one class with different membership values

Linguistic variables (LVs) are the input or output variables of the system. LVs values are words or sentences from a natural language, instead of numerical values. A linguistic variable values consist of a set of linguistic terms. For example, temperature values can be described by linguistic terms as; too cold, cold, warm, hot and too hot, these terms describe a specific feature of the temperature and it is expressed as:

$T(t)=\{$ too cold, cold, warm, hot, too hot $\}$

It is common to use words like short, medium and tall, or low, medium and high, to describe some fuzzy features. In case of image processing, linguistic variable can be any of the image features like luminance, colour, brightness etc. the values for each of these features can be described in terms of linguistic terms like; bright, dark and very dark.

Figure 3 shows the difference between crisp membership function and fuzzy membership function, in crisp the output value of the function is either one or zero, while the fuzzy membership function maps the element to a real value between 0 and 1 . Various types of membership functions are used like triangular, trapezoidal, generalized bell shaped, Gaussian curves, polynomial curves, and sigmoid functions.

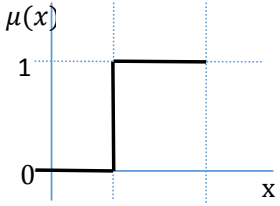

(a)

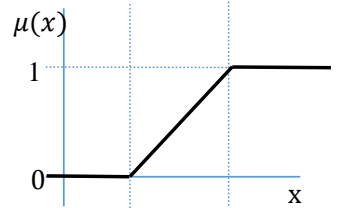

(b)
Figure 3 Crispy vs. Fuzzy classification, (a) crisp membership function; (b) fuzzy membership function.

\subsection{Fuzzy Based Image Processing}

The vagueness in pixel luminance value results in a noncrispy definition of the features in an image. Thus, fuzzy definition is required in image feature extraction like in edge detection and segmentation. The incertitude in an image maybe defined in terms of greyness ambiguity or spatial ambiguity or both of them. Greyness ambiguity can be defined as the indefiniteness in deciding whether the pixel is white or black while spatial ambiguity, which is also known as geometrical ambiguity may arise in shape or geometry like finding the centroid, shape edges etc. of a region.

The image $I$ of size of $M \times N$ can be represented as an array of fuzzy singletons; each member in this array has a value of membership denoting its degree of possessing some property like brightens darkness, etc. It can be represented in fuzzy notation as:

$I=\left\{\mu_{x}\left(x_{m n}\right): m=1,2, \ldots, M ; n=1,2, \ldots, N\right\}$

Where $\mu_{x}\left(x_{m n}\right)$ is the degree of possessing such property $\mu$ by the $(m, n) t h$ pixel and can be defined using global, local or spatial information

\section{THE PROPOSED ALGORITHIM}

As it was explained earlier, bimodal image can be divided into two distinct regions by selecting appropriate thresholding value at which separate the object from its background.

If $I(x, y)$ is an image and $B(x, y)$ is the binary image after thresholding then:

$$
B(x, y)= \begin{cases}1, & \text { if } I(x, y) \geq T \\ 0, & \text { if } I(x, y)<T\end{cases}
$$


Where $T$ is the thresholding value. One (1) represents a pixel on the object and zero (0) represents the background.

Finding $\mathrm{T}$ is a crucial issue and represents the main problem in such algorithms. Many algorithms have been proposed such as local threshold, global threshold, and adaptive threshold values extraction. Genetic algorithms, neural networks, Gaussian, and other techniques are also used. Most of the techniques consider the Histogram to find the threshold values. One of the most popular techniques is the bimodal histogram splitting, in which the histogram is split into two regions, light and dark regions, or white and black in crisp set definition.

As shown in Figure 4, which represents the image thresholding based on the histogram valley selection, the crisp definition for the pixels with luminance values less than $\mathrm{T}$ is black always while the values above $\mathrm{T}$ are white. It is also clear from the figure above that the value of $\mathrm{T}$ is not the minimum value of the histogram but it should be the minimum value between two peaks.

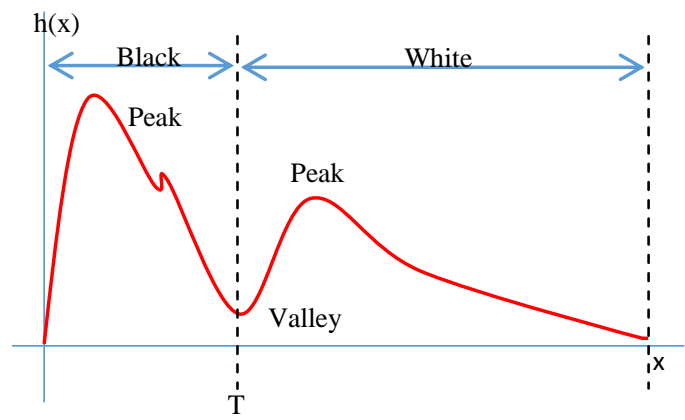

Figure 4 Bimodal Histogram Thresholding

Base on the fuzzy intelligence theory this is not the actual case because here it was considered that there is a welldefined borders between the object and its background.

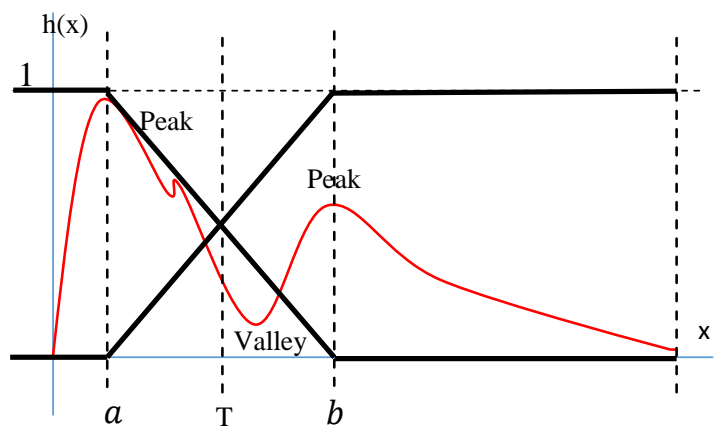

Figure 5 Bimodal Fuzzy Histogram Thresholding

As shown in Figure 5 the histogram is approximated into two membership functions; $\mu_{b}(x)$ which measure how much is the pixel black, and $\mu_{w}(x)$ which measure the white membership value. Thus, the luminance space will be divided into two subspaces or subsets $\mathrm{B}$ and $\mathrm{W}$. According this figure the histogram is divided into 3 region rather than 2 regions as in crisp thresholding. One of the regions is the black region and the second one is the white region. The third region, which is the overlapping between the black and white regions, has been generated due to the uncertainty in image luminance value and it may represent the fading or the shadow. The value of $\mathrm{T}$ can be found in different ways of which are:
1. The intersection of the two lines of the membership functions.

2. The value of $\mathrm{T}$ can be extracted by calculating the entropy of the regions.

3. Using AI techniques like Genetic Algorithms and Neural Networks.

The membership function can be derived from the figure as follows:

$m_{w}=\frac{1}{a-b} ; \quad \mu_{w}(x)=\frac{1}{a-b} x+c ; 0=\frac{1}{a-b} b+c$

$c=\frac{-b}{a-b} ; \mu_{w}(x)=\frac{1}{a-b} x-\frac{b}{a-b}$

$\mu_{w}(x)=\left\{\begin{array}{cc}1 & x<a \\ \frac{x-b}{a-b} & a \leq x<b \\ 0 & x \geq b\end{array}\right.$

In the same way:

$$
\mu_{b}(x)=\left\{\begin{array}{lr}
0 & x<a \\
\frac{a-x}{a-b} & a \leq x<b \\
1 & x \geq b
\end{array}\right.
$$

\section{TESTING AND RESULTS}

The proposed method has been applied on grey images, for colour images the thresholding algorithm is applied three times, one for each band (R, G, and B). It starts with histogram extraction from a grey image. The extracted histogram may contain some sharp changes which occurred due to abnormal values in the histogram, thus one dimensional low pass filter (LPF) is applied. This LPF results in a smooth histogram. The peaks and valley are extracted from the histogram using one-dimensional discrete gradient.

The membership functions are extracted based on the extracted peaks. The membership functions intersection points (MFIP) which shall be used as the threshold value can be obtained from the extracted membership functions by finding the intersection between them.

The common region between the white and black regions can be extracted using (if-then) rues, i.e.

$$
\text { If } x \in B \text { and } x \in W \text { then } x \in \text { (common region) }
$$

This can be found also using the formula $\mu_{A \cap B}(x)=$ $\operatorname{Min}\left(\mu_{A}(x), \mu_{B}(x)\right)$, which represents the ANDing process between the two regions.

The common region between the object and its background may represent the shadow of the object or the transition from the object to the background.

The above algorithm has been applied on different types of images as shown in Figure 6. The histogram shown is approximated to two membership functions, by which the membership value of each pixel is calculated. According to the membership value, the pixel is decided whether it is black, white or grey in some level. The grey value can be considered as the intersection between the two membership functions. In Figure 6 the first image was thresholded using bimodal technique $(T=205)$ from which the objects were separated from the background but still the holes in the images are ambiguous whether they belong to the background or to the object. By applying membership functions intersection point MFIP $(T=124)$ gives better results in separating the objects. The difference between the white and black or the common region that belongs to both membership functions is given the 
last column. This might occur due to the ambiguity in the greyness or spatial.

\section{CONCLUSIONS}

As a conclusion to the above discussion, one may consider several points as will be explained here. The algorithm is suitable for images with bimodal grey level distribution and gave excellent results when it was applied on such kind of images. The histogram smoothing gave strength to the method because it overcomes the local maxima and minima due to the abnormal values in the histogram. The used method can be modified to be applicable with non-bimodal images by repeating the operation more than one time. Also preprocessing may improve the results, thus different kind of reprocessing can be used such as image smoothing and histogram smoothing. The proposed method does not need that much calculations that are needed in other applications since it is not an iterative process. In addition, the obtained results are far better than those obtained by applying the crispy techniques.

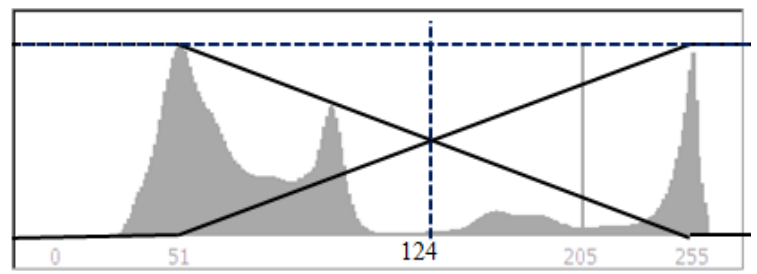

(a)

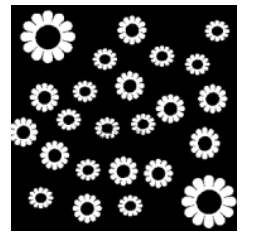

(b)

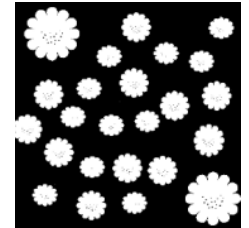

(c)

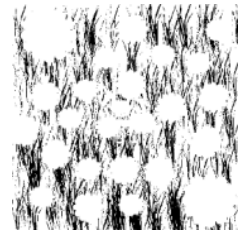

(d)
Figure 6 Applying the Fuzzy-Bimodal Thresholding Algorithm. (a) Histogram representation with fuzzy membership function, (b) traditional bimodal thresholding results, (c) applying FBT results, and (d) the common region between the object and its background

\section{REFERENCES}

[1] "Wikipedia / hsl and hsv," [online]. Available: http://en.wikipedia.org/wiki/hsl_and_hsv. [accessed 19 2011].

[2] Koschan and m. Abidi, digital color image processing, new jersey: john wiley \& sons, inc., 2008.

[3] W. K. Pratt, digital image processing, 4th. Ed., hoboken, new jersey: john wiley \& sons, inc., 2007.

[4] W. Skarbek and a. Koschan, colour image segmentation a survey, sekretariat fr 5-4: leiter der fachbibliothek informatik, 1994.
[5] S. Thilagamani and n. Shanthi, "a survey on image segmentation through clustering," international journal of research and reviews in information sciences, vol. 1, no. 1, pp. 14-17, march 2011.

[6] T. N. Pappas, "an adaptive clustering algorithm for image segmentation," ieee transactions on signal processing, vol. 10, no. 1, pp. $901-914,1992$.

[7] K. Deshmukh and g. N. Shinde, "an adaptive neuro-fuzzy system for color image segmentation," j. Indian inst. Sci., no. 8, p. 493-506, sept.-oct. 2006.

[8] J. Shi and j. Malik, "normalized cuts and image segmentation," ieee transactions on pattern analysis and machine intelligence,, vol. 22 , no. 8 , august 2000

[9] H. D. Cheng, j.-r. Chen and j. Li, "threshold selection based on fuzzy c-partition entropy approach," pattern recognition, vol. 31, no. 7, pp. 857-887, 1998.

[10] Q. Wang, z. Chi and r. Zhao, "image thresholding by maximizing the index of nonfuzziness of the 2-d grayscale histogram," computer vision and image understanding, vol. 85, p. 100-116, 2002.

[11] W.-b. Tao, j.-w. Tian and j. Liu, "image segmentation by three-level thresholding based on maximum fuzzy entropy and genetic algorithm," pattern recognition letters, no. 24, pp. 3069-3078, 2003.

[12] Y. Yong, . Z. Chongxun and . L. Pan, "a novel fuzzy cmeans clustering algorithm for image thresholding," measurement science review, vol. 4, no. 1, 2004

[13] T. Junwei, h. Yongxuan and y. Yalin, "fuzzy c-means cluster image segmentation with entropy constraint," in the 33rd annual conference of the ieee industrial electronics society (iecon), taipei, taiwan, nov. 5-8, 2007.

[14] H. R. Tizhoosh, "image thresholding using type ii fuzzy sets," pattern recognition, no. 38 , pp. 2363-2372, 2005.

[15] M. S. Prasad and e. Al, "unsupervised image thresholding using fuzzy measures," international journal of computer applications, vol. 27, no. 2, pp. 32-41, 2011.

[16] L. A. Zadeh, "fuzzy sets," information and control, vol. 8, pp. 338-353, 1965

[17] A. Alshennawy and a. A. Aly, "edge detection in digital images using fuzzy logic technique," world academy of science, engineering and technology, vol. 51, pp. 178$186,2009$.

[18] D. Kulkarni, computer vision and fuzzy-neural systems, saddle river, nj, usa: prentice hall, 2001.

[19] M. Al-azawi and n. K. Ibrahim, "bimodal histogram based image segmentation using fuzzy-logic," in ncaiae12, massana, 2012. 